\title{
Risk Stratification for Acute Kidney Injury in Critically-ill Children
}

\author{
SidHARTH KUMAR SETHI ${ }^{1}$ AND RUPESh RAINA ${ }^{2}$ \\ From ${ }^{1}$ Pediatric Nephrology, Kidney Institute, Medanta, The Medicity, Gurgaon, Haryana, India; \\ and ${ }^{2}$ Pediatric Nephrology,Akron Children's Hospital,Akron,Ohio,USA. ${ }^{1}$ sidsdoc@gmail.com
}

1 cute Kidney Injury (AKI) is common in critically-ill children; the prevalence varies from $10 \%$ to $82 \%$ depending on the underlying disease severity [1,2]. Increasing severity of AKI, based on serum creatinine and urine output, is associated with an increased mortality in adults and children $[3,4]$. Unfortunately, using serum creatinine as a marker for AKI has multiple limitations, including a delayed rise and a loss of kidney function by the time kidney injury is recognized, and this may delay the intervention needed [5].

There has been an extensive research on the utility of biomarkers for identification and prediction of AKI in children. These have been validated in cardiopulmonary bypass surgery setting, where timing of ischemic insults and lack of comorbidities is known [6]. However, children who present to the non-cardiac intensive care unit (ICU) have demographic heterogeneity and multiple comorbidities, including septicemia, shock and multiorgan dysfunction.

Estimation of troponin in acute coronary syndrome is a classic example of a directed, optimized biomarker testing. Troponin testing in a patient with cardiac angina, clinical signs and known risk factors helps the clinician to rule out myocardial infarction in the triage itself. In the selected risk stratified population, troponin has high specificity and positive predictive value. Dr Chawla and Dr Goldstein proposed a conceptual model, termed 'renal angina' to identify critically-ill patients at the risk for AKI at the time of ICU admission. Renal Angina Index (RAI) aims to delineate patients at risk of subsequent AKI using patient demographic factors and early signs of kidney injury [7]. In the initial multicentric cohort study, Basu, et al. [7] demonstrated that patients with RAI of 8 or more had higher rates of AKI on day 3 of admission with an area under the curve (AUC) to predict being 0.74-0.81. RAI outperformed traditional markers of illness severity (Pediatric Risk of Mortality-II), and 'Kidney Disease Improving Global Outcomes' (KDIGO) staging used to classify AKI.
Especially in the developing world, utilization of RAI may be beneficial in identification of sick children 'at-risk' of early AKI, and in the judicious use of biomarkers, daily biochemical assessment and providing specialized renal care to children with higher RAI. There have been multiple studies on the validation of RAI in critical children. In an earlier study from India, we demonstrated that RAI was better than traditional markers of AKI and illness severity scores with high AUC in RAI $\geq 8$, and higher negative predictive values for RAI $<8$ [8]. The study [9], published in the current issue of Indian Pediatrics, adds on to the evidence that day 0 RAI has a good sensitivity, specificity, and negative predictive value for AKI on day 3 of admission. A recent study from New Delhi [10] added that RAI thresholds $\geq 12$ or $\geq 20$ had higher specificities, Youden index, and positive predictive value than that of $\mathrm{RAI} \geq 8$ in discriminating severe $\mathrm{AKI}$ on day 3 or 7, and distinguished between patients with and without need for dialysis.

RAI is a simple and clinically feasible index, which can be done bedside to identity high-risk patients. It may also help the clinician to decide which patient may benefit by biomarker assessment. RAI uses simple identifiable criteria (e.g., a child on inotropes), and helps in identification of subsequent AKI. Patients with a higher RAI may be subjected to intense monitoring, preventing fluid overload, avoiding nephrotoxins, early initiation of renal replacement therapy, and more comprehensive directed renal care. These RAI-positive children may serve as a uniform group, who can be enrolled in clinical biomarker and therapeutic trials.

There is a need for large, multicentric pediatric ICU databases, to refine the cut-off of RAI, rigorous evaluation of the risk factors to be put in the index and evidence of renal injury. This will allow for validation and calibration of RAI in the high-risk population. Indian Society of Pediatric Nephrology currently has a Chronic Kidney Disease Registry, and is working on Neonatal AKI Consortium, and plans to add on AKI epidemiology study soon. 
EDITORIAL

To conclude, risk stratification using RAI can definitely aid a clinician and intensivist for prediction of severe AKI, and to reliably differentiate children who shall respond to conservative therapy or require specialized renal care.

Funding: None; Competing interests: None stated.

\section{REFERENCES}

1. Schneider J, Khemani R, Grushkin C, Bart R. Serum creatinine as stratified in the RIFLE score for acute kidney injury is associated with mortality and length of stay for children in the pediatric intensive care unit. Crit Care Med. 2010;38:933-9.

2. Akcan-Arikan A, Zappitelli M, Loftis LL, Washburn KK, Jefferson LS, Goldstein SL. Modified RIFLE criteria in critically ill children with acute kidney injury. Kidney Int. 2007;71:1028-35.

3. Srisawat N, Hoste EE, Kellum JA. Modern classification of acute kidney injury. Blood Purif. 2010;29:300-7.

4. Slater MB, Anand V, Uleryk EM, Parshuram CS. A systematic review of RIFLE criteria in children, and its application and association with measures of mortality and morbidity. Kidney Int. 2012;81:791-8.

5. Goldstein SL. Acute kidney injury biomarkers: renal angina and the need for a renal troponin I. BMC Med. 2011;9:135.

6. Han WK, Waikar SS, Johnson A, Betensky RA, Dent CL, Devarajan $\mathrm{P}$, et al. Urinary biomarkers in the early diagnosis of acute kidney injury. Kidney Int. 2008;73:863-9.

7. Basu RK, Zappitelli M, Brunner L, Wang Y, Wong HR, Chawla LS, et al. Derivation and validation of the renal angina index to improve the prediction of acute kidney injury in critically ill children. Kidney Int. 2014;85:659-67.

8. Sethi SK, Raghunathan V, Shah S, Dhaliwal M, Jha P, Kumar M, et al. Fluid overload and renal angina index at admission are associated with worse outcomes in critically ill children. Front Pediatr. 2018;6:118.

9. Gawadia J, Mishra K, Kumar M, Saikia D. Prediction of severe acute kidney injury using renal angina index in a pediatric intensive care unit. Indian Pediatr. 2019;56: 647-52.

10. Sundararaju S, Sinha A, Hari P, Lodha R, Bagga A. Renal angina index in the prediction of acute kidney injury in critically ill children. Asian J Pediatric Nephrol. 2019;2: 25-30. 